\title{
Structural Characterization of a Cation-selective, Self-assembled Peptide Pore in Planar Phospholipid Bilayers
}

\author{
Evelyne Deplazes $^{\mathrm{a}^{* \#}}$, Lissy M. Hartmann ${ }^{\mathrm{a}^{*}}$, Charles G Cranfield ${ }^{\mathrm{a}}$, Alvaro Garcia ${ }^{\mathrm{a} \#}$ \\ *joint first authors \\ \# corresponding authors
}

aSchool of Life Sciences, University of Technology, Sydney, Ultimo, NSW 2007, Australia.

\begin{abstract}
GALA is a 30-residue, amphipathic peptide that self-assembles into multimeric, transmembrane pores in a $\mathrm{pH}$-dependent fashion. In this study, we characterize the size, multimeric structure and cation selectivity of GALA pores in planar phospholipid bilayers using electrical impedance spectroscopy and molecular dynamics simulations. We demonstrate that in planar bilayers GALA pores are likely formed by six peptide monomers rather than eight to twelve monomers as previously reported in lipid vesicles. Besides, we show that in planar bilayers GALA pores exhibit previously unreported cation selectivity. We propose that the difference in predicted pore structure between planar bilayers and lipid vesicles exemplifies the importance of phospholipid bilayer structural properties on the aggregation of transmembrane helical structures.
\end{abstract}

Keywords: viral fusion peptides, peptide-membrane interactions, molecular dynamics simulations, phospholipid bilayers, membranes, curvature 


\section{Author final version}

In this letter, we demonstrate that the structure of multimeric, transmembrane pores formed by the peptide GALA in planar bilayers is different from the reported structure in lipid vesicles. In the previous studies ${ }^{1}$, using data from leakage assays, GALA pores were estimated to consist of 8-12 peptide monomers in unilamellar lipid vesicles (LUVs, $100 \mathrm{~nm}$ ). Here we report that in planar lipid bilayers GALA pores are most likely composed of 6 monomers and exhibit ion selectivity based on cation hydration energies. Numerous studies investigate membrane curvature on how proteins interact with membranes, including protein recruitment, aggregation, localization, and curvate 'sensing' ${ }^{2-8}$. However, the effect of mesoscale properties such as lipid bilayer asymmetry on the quaternary structure of transmembrane peptides is poorly understood and understudied.

GALA is a 30-residue peptide (WEAALAEALAEALAEHLAEALAEALEALAA), designed by Subbarao et $\mathrm{al}^{9}$ to mimic the membrane insertion activity of viral fusion peptides, with insertion triggered by acidic $\mathrm{pH}^{10-11}$. The repeat sequence of glutamic acid (Glu, E), alanine (Ala, A) and leucine (Leu, L) provide a hydrophobic sequence EAL with strong helical propensity $^{12-13}$ while the titratable Glu side chain is responsible for the $\mathrm{pH}$-dependent conformational change. At neutral $\mathrm{pH}$ GALA is unstructured. At acidic $\mathrm{pH}(\mathrm{pH}<6)$, the protonation of the Glu residues induces a conformational change to an $\alpha$-helix ${ }^{9,14}$. The poreforming and membrane-disrupting activity of GALA has been assessed in several studies using phospholipid vesicles and fluorescence leakage assays 1,9,15-17. Maximal leakage was detected at acidic $\mathrm{pH}$ values $(\mathrm{pH}<6.0)$ and increasing the peptide:lipid ratio also increased leakage ${ }^{1}$, ${ }^{15}$. Parente et al. ${ }^{1}$ developed a theoretical model that describes the kinetics of GALA-induced leakage as a bimodal pore-forming mechanism. Initially, individual peptides bind to the membrane surface where they aggregate. Once aggregates reach a critical size, peptides insert into the membrane to form stable pores, resulting in leakage. The theoretical model was fitted to experimental leakage data varying the fraction of peptides bound, the forward rate of aggregation and the number of peptides that form a pore. From this model, as well as data from leakage assays with different sized molecules, the authors ${ }^{1}$ proposed that GALA "forms a transbilayer channel composed of 8-12 monomers". In a later study, Nicol et al ${ }^{15}$ demonstrate this model can reproduce GALA-induced leakage from cholesterol-containing vesicles.

This proposed model assumes a static, barrel-stave like pore formed by rigid, rod-like helices and provides little direct structural information. As such, this model ignores the inherent 


\section{Author final version}

flexibility of helical peptides in a fluid membrane or the effect of membrane structure on the pore structure. In this study, we combine tethered lipid bilayer membranes (tBLM) and swept frequency electrical impedance spectroscopy (EIS) with molecular dynamics (MD) simulations to characterize the structure of GALA pores in planar phospholipid bilayers.

In tBLMs, a phospholipid bilayer is formed on a gold substrate by incorporating a small fraction of hydrophobic anchor molecules in the inner leaflet of a lipid bilayer. The majority of the lipids in the inner leaflet and all of the lipids in the outer leaflet are thus mobile, mimicking the fluidity of cell membranes (Fig S1A). EIS uses an AC frequency sweep to determine the impedance caused by the phospholipid bilayer, which is fitted to an equivalent circuit (Fig S1B) to obtain a measure of membrane conductivity. This enables tBLM/EIS to be used to monitor real-time membrane permeability of ions ${ }^{18}$. To get an upper limit of the conductivity of GALA pores in planar membranes, we carried out tBLM/EIS measures in the presence of organic cations tetraethylammonium (TEA, MW $130 \mathrm{~g} / \mathrm{mol}$ ) and choline (MW 104 $\mathrm{g} / \mathrm{mol}$ ) (Fig S2). The cations TEA and choline are of similar size to the cation 8-amino naphthalene-1,2,3-trisulfonic acid (ANTS, MW $445 \mathrm{~g} / \mathrm{mol}$ ) (Fig S2), which was the permeant in the leakage assays by Parente et $\mathrm{al}^{1}$. After confirming the formation of stable ion-conducting GALA pores (Fig. S3) in phosphatidylcholine (POPC) membranes, increasing concentrations of choline.Cl or TEA.Cl was added to the POPC-tBLMs in the presence and absence of GALA (Fig 3A, B). The absolute conductance depends on the number of GALA pores present, which cannot be controlled in our experiments. Thus, a comparison of the conduction slopes from the different ions necessitates normalization to the initial membrane conduction obtained at $25 \mathrm{mM}$ for each cation tested. This provides a quantitative measure of the ability of GALA pores to conduct ions (Fig 3C). As seen in Fig 3, there is no concentration-dependent increase in the membrane conduction for choline or TEA, indicating that these cations are unable to pass through the GALA pore. 


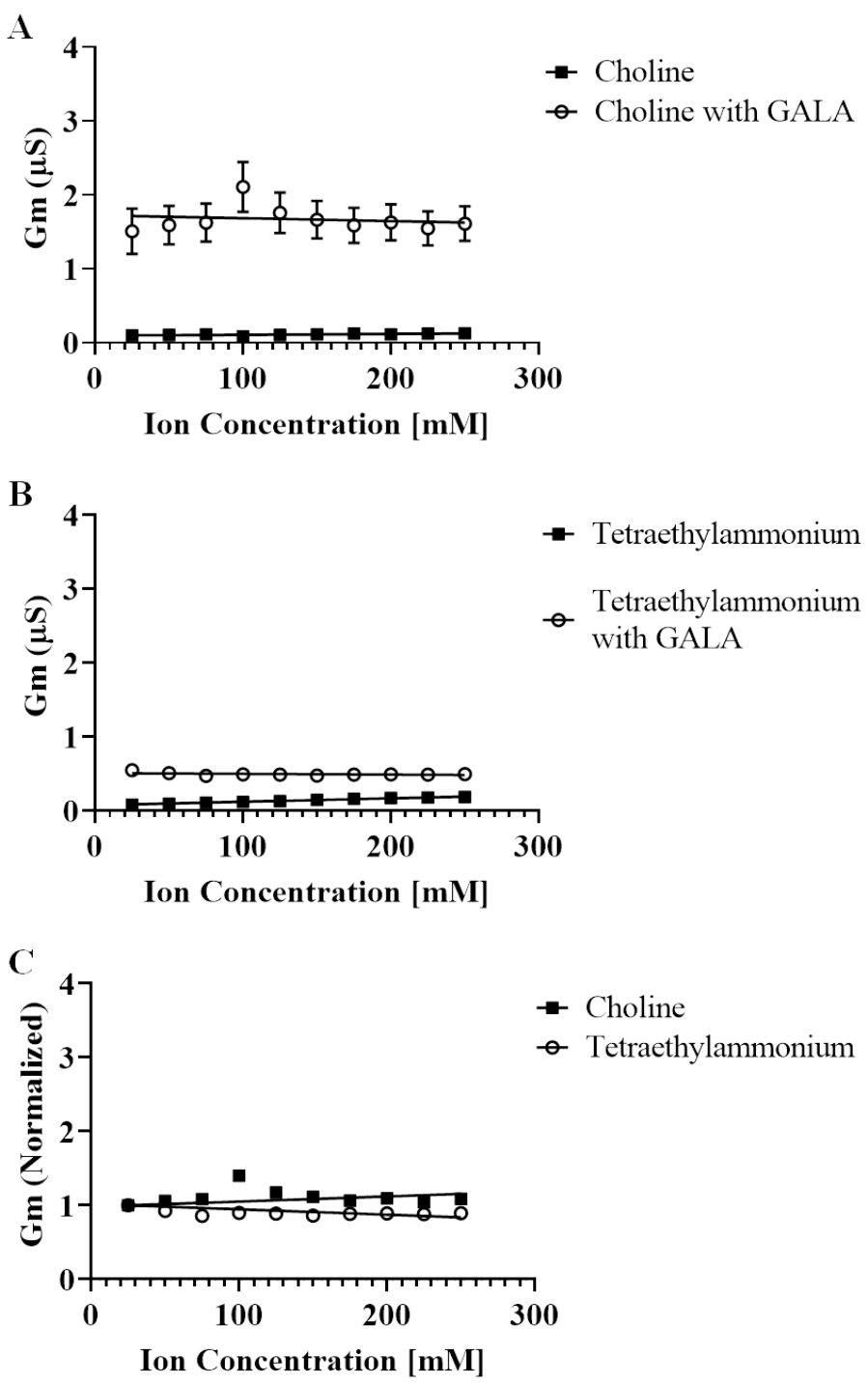

Fig. 1. tBLM/EIS measurements of conductance for the organic cations choline and Tetraethylammonium across POPC bilayers with GALA ( $\square$ ) and without GALA (•) at pH 55 ( $\mathrm{n}=6$, otherwise mentioned). (A) Absolute change in conductance with increasing concentration of choline. (B) Absolute change in conductance with increasing concentration of Tetraethylammonium. (C) Relative change in conductance with increasing concentration of choline and Tetraethylammonium. Errors represent \pm standard error of the mean from replicates. Error bars are absent when error bars are smaller than the symbol. All measurements were taken at room temperature.

The inability of choline to pass through the GALA pore in the bilayer would indicate that the pore in our planar bilayer is much smaller than the pore size estimated from leakage assays in vesicles. To provide structural information, we carried out a series of MD simulations of GALA 


\section{Author final version}

pores formed by either six, seven or eight peptide monomers embedded into POPC membranes, and solvated with choline and $\mathrm{Cl}^{-}$ions. These simulation systems are referred to as Hex, Hept and Oct, respectively. For each system, a sequence of position restrained simulations was carried out, followed by a 400-ns production run. For all systems, a stable pore formed after approximately 200 ns, as assessed by time-dependent Root Mean Square Deviation data (Fig S4A). Clustering analysis was used to ensure that all structures analyzed are representative of conformations sampled in a given simulation.

Most helices are slightly tilted concerning the bilayer normal to match the hydrophobic thickness of the phospholipid bilayer (Fig S5), and all three systems show stable, water-filled pores (Fig. 2). Besides, most helices deviate from an ideal, straight helix and their transmembrane alignment or tilt varies. As a result, the GALA pores observed in the simulations differ from the idealized polygons used to estimate pore sizes. The pores contain the following average number of water molecules: Hex $26 \pm 3$; Hept $145 \pm 10$; Oct $149 \pm 9$, suggesting that Hex pores contain significantly fewer water molecules than Hept and Oct pores.

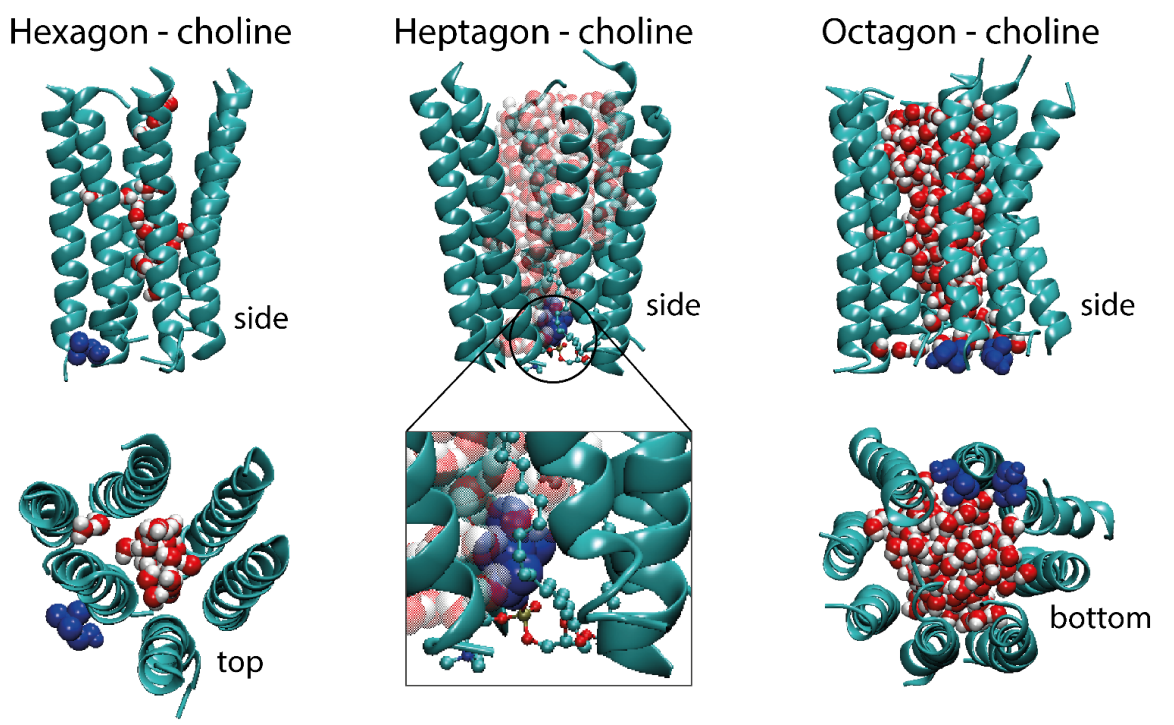

Fig 2: Structure of GALA pores in the presence of choline chloride. Representatitive structures of Hexagon (Hex), Heptagon (Hept), and Octatong (Oct) pores obtained from the last $100 \mathrm{~ns}$ of a 400-ns MD simulation of GALA pores in the presence of choline chloride. Side view refers to structures viewed parallel to the membrane normal. Top and bottom view refer to structures viewed perpendicular to the membrane normal. Peptides are shown in cartoon representation (cyan). Water molecules (red-white) inside the pore and choline ions are shown as van der 
Waals spheres (red-white and blue, respectively). Lipids and water outside the pore are omitted for clarity.

The simulations suggest that both Oct and Hept pores are large enough for choline to pass through the channel. In the Hept pore, the choline ion is observed to enter the pore despite parts of the pore being obscured by a lipid molecule. In contrast, the Hex pores are likely too small for choline to pass through. We repeated simulations of Hex, Hept, and Oct pores in the presence of $\mathrm{NaCl}$ using the same approach as for simulations with choline chloride (Fig 3). As before, stable pores were formed after $\sim 200 \mathrm{~ns}$ of unrestrained MD simulations (Fig S4B). No discernible difference in the overall structure of pores in the presence of $\mathrm{NaCl}$ was observed. As before, the Hept and Oct pores contain significantly more water than the Hex pores (Hex $16 \pm 3$; Hept $110 \pm 9$; Oct $161 \pm 14$ ). $\mathrm{Na}^{+}$ions were observed to pass the Hept, or Oct pores fully solvated, and for Oct pores, several $\mathrm{Na}^{+}$ions can be present inside the pore simultaneously (Fig. 3). Similarly, simulations of Hex, Hept, and Oct pores in the presence of $\mathrm{CaCl}^{2}$ showed that $\mathrm{Ca}^{2+}$ ions could permeate Hept and Oct pores in a fully solvated state (Fig. S6).

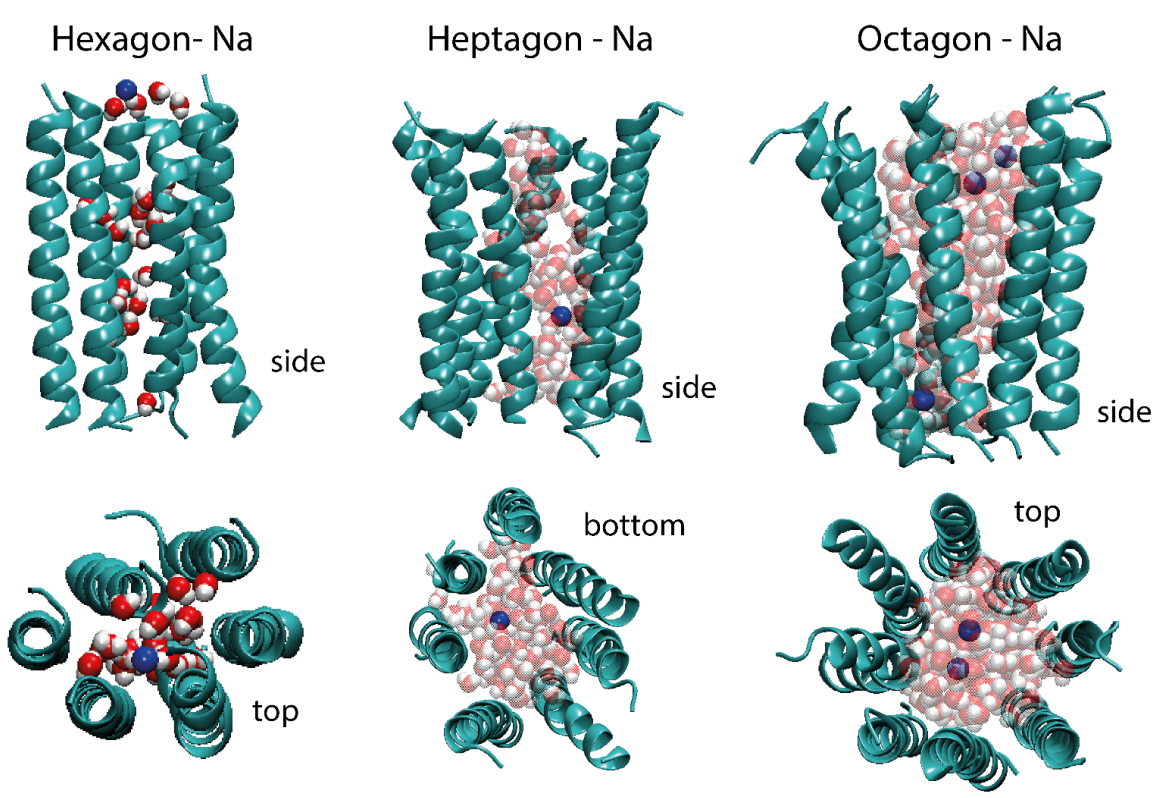

Fig 3: Structure of GALA pores in the presence of $\mathrm{NaCl}$. Representatitive structures of Hexagon (Hex), Heptagon (Hept), and Octatong (Oct) pores obtained from the last $100 \mathrm{~ns}$ of a 400-ns unrestrained MD simulation of GALA pores in the presence of $\mathrm{NaCl}$. Side view refers to structures viewed parallel to the membrane normal. Top and bottom view refer to structures viewed from along the membrane normal. Peptides are shown in cartoon representation (cyan). Water molecules inside the pore are shown as van der Waals spheres (red-white), and sodium 


\section{Author final version}

ions are shown as blue van der Waals spheres. Lipids and water outside the pore are omitted for clarity. Images of the pores in the membrane environment can be found in Fig S5 in the supplementary data.

The MD simulations suggest that in planar membranes the Hept and Oct are large enough for $\mathrm{Na}^{+}$ions to pass through the pore in a fully solvated state but have to be partially desolvated to pass through a hexameric GALA pore. The free energy of hydration $\left(\mathrm{G}_{\text {hydration }}\right)$ for monovalent cations is significantly less negative (i.e. more favorable) than for divalent cations ${ }^{19}$ (Table S1).

If cations need to partially desolvate to pass through hexameric GALA pores in planar membranes, we would expect that the concentration-dependent change in conductance would be significantly different for mono- and divalent cations. This would be reflected in different slopes in the conductance vs concentration data. To test this, we repeated tBLM/EIS experiments with mono- and divalent cations. For this, increasing concentrations of the monovalent cations $\mathrm{LiCl}, \mathrm{NaCl}$ and $\mathrm{KCl}$, and divalent cations $\mathrm{CaCl}_{2}, \mathrm{MgCl}_{2}$ and $\mathrm{SrCl}_{2}$ were added to POPC-tBLMs in the presence and absence of GALA. Measurements were conducted at $\mathrm{pH}$ five and ion concentrations, as chloride salts, were increased from $25 \mathrm{mM}$ to $250 \mathrm{mM}$ using $25 \mathrm{mM}$ increments. As a control, the same titration was conducted in the absence of GALA peptide where minimal background membrane conduction was observed. There is a linear, concentration-dependent increase in membrane conduction with increasing ion concentration for all mono- and divalent cations (Fig S7). As before, to enable comparison between different ions, conductance data were normalized to the initial membrane conduction obtained at $25 \mathrm{mM}$ for each cation tested. Fig. 4 shows comparisons of normalized conductance for monovalent and divalent cations, respectively. 

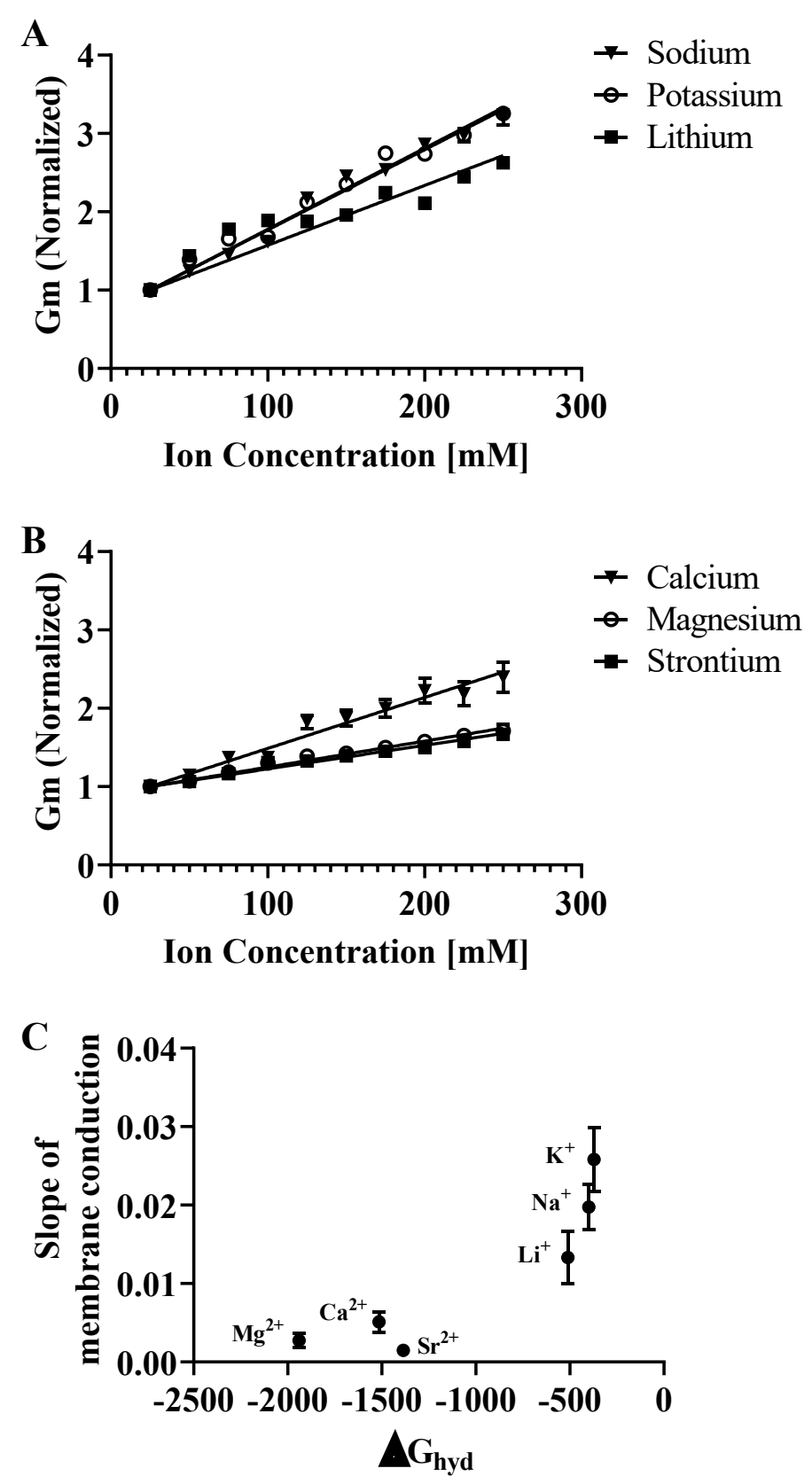

Fig 4. tBLM/EIS measurements of conductance across POPC bilayers in the presence of GALA comparing three different ion types at $\mathrm{pH}$ 5. (A) Relative change in conductance with increasing concentration of monovalent alkali cations lithium, sodium and potassium. (B) Relative change in conductance with increasing concentration of divalent alkaline cations magnesium, calcium, and strontium. (C) Mean of the slope gradients plotted against $\Delta \mathrm{G}_{\text {hydration. }}$. Error bars represent \pm standard error of the mean from replicates. Error bars are absent when error bars are smaller than the symbol. All measurements were taken at room temperature. (n $=6$, otherwise mentioned) 
The slope of the normalized, concentration-dependent membrane conduction provides us with some information of the selective ion permeability of the GALA pore with a steeper slope indicating a higher ion permeability. Comparing the normalized slopes for $\mathrm{Li}^{+}, \mathrm{Na}^{+}$and $\mathrm{K}^{+}(\mathrm{Fig}$ 4A) show no statistical difference between $\mathrm{Li}^{+}, \mathrm{Na}^{+}$and $\mathrm{K}^{+}$. No difference was observed between the alkaline earth metals tested (See Fig. 4B for $\mathrm{Ca}^{2+}, \mathrm{Mg}^{2+}$ and $\mathrm{Sr}^{2+}$ ). However, all of the alkaline earth metal slopes were significantly lower than $\mathrm{K}^{+}$and $\mathrm{Na}^{+}$, and the difference between $\mathrm{Li}^{+}$and $\mathrm{Ca}^{2+}$ was not significant. When the gradient of the slopes was compared against $\Delta \mathrm{G}_{\text {hydration }}$ energy of the cations (Table S1) a trend is observed suggesting desolvation energies are a determining factor for ion conduction through the GALA pore (Fig. 4 C).

Our data from tBLM/EIS and simulations suggest that GALA forms smaller pores in planar membranes compared to vesicles. Specifically, the smaller Hex pore, which contains significantly fewer water molecules and is capable of only a modest desolvation energy-based ion selectivity. More interestingly, our data indicate that the structure and size of GALA pores are different in LUVs compared to planar membranes. The data from two previous studies ${ }^{1}$, 15 clearly shows that ANTS leaks through pores in vesicles. In contrast we report that TEA and choline, which are of similar size to ANTS, do not leak through pores in planar membranes. The experimental conditions in previous experiments and the ones reported here are comparable with respect to phospholipids used, $\mathrm{pH}$ and ionic strengths of buffers. The most obvious difference is the use of planar bilayers vs LUVs. While curvature itself may be minimal in LUVs, several studies have reported that LUVs composed of zwitterionic phospholipids exhibit differences in lipid packing, area per lipid, hydration or charge distribution ${ }^{21-24}$ caused by asymmetry between the inner and outer bilayer leaflets. This asymmetry may impart distortive forces on GALA pores that change the aggregation of the monomers and thus affect

pore size. The increased lipid packing ${ }^{25-26}$ and increased order of interfacial water ${ }^{27}$ caused by low $\mathrm{pH}$, might further exassabated the asymmetry.

Finally, GALA provides a simple and tunable model system to study the effect of lipid bilayer asymmetry on the structure of transmembrane peptide pores, and more generally the spatial arrangement of transmembrane helices; an impact that is poorly understood.

\section{Acknowledgement}

The authors would like to acknowledge Associate Prof. Ronald Clarke, University of Sydney and Dr Bruce Cornell for their helpful feedback in the preparation of this manuscript. A.G. and 
E.D. are funded by the UTS Chancellor's Postdoctoral Research Fellowship scheme. This work was supported by resources provided by the Pawsey Supercomputing Centre with funding from the Australian Government and the Government of Western Australia and computational resources provided by the UTS eResearch High-Performance Computer Cluster.

\section{Supporting Information}

The supporting information contain details of the methods for the tBLM/EIS experiments and all MD simulations and additional results. These include the structure of the organic cations choline, TEA and ANTs, as well as a Table listing the ionic radii and solvation energies of cations used in this study. Additional results include data showing the formation of stable GALA pores in membranes at $\mathrm{pH}$, the RMSD vs time graphs for demonstrating the stabilyt of pores in the MD simulations, the structure and position of of these pores in a membrane environment, structure of GALA pores in the presence of $\mathrm{CaCl}_{2}$.

\section{References}

1. Parente, R. A.; Nir, S.; Szoka, F. C., Mechanism of leakage of phospholipid vesicle contents induced by the peptide GALA. Biochemistry 1990, 29 (37), 8720-8728.

2. Aimon, S.; Callan-Jones, A.; Berthaud, A.; Pinot, M.; Toombes, G. E.; Bassereau, P., Membrane shape modulates transmembrane protein distribution. Developmental Cell 2014, 28 (2), 212-8.

3. Attard, G. S.; Templer, R. H.; Smith, W. S.; Hunt, A. N.; Jackowski, S., Modulation of CTP:phosphocholine cytidylyltransferase by membrane curvature elastic stress. Proc Natl Acad Sci U S A 2000, 97 (16), 9032-9036.

4. Brown, M. F., Curvature Forces in Membrane Lipid-Protein Interactions. Biochemistry 2012, 51 (49), 9782-9795.

5. Hatzakis, N. S.; Bhatia, V. K.; Larsen, J.; Madsen, K. L.; Bolinger, P.-y.; Kunding, A. H.; Castillo, J.; Gether, U.; Hedegård, P.; Stamou, D., How curved membranes recruit amphipathic helices and protein anchoring motifs. Nature Chemical Biology 2009, 5 (11), 835-41.

6. Kurylowicz, M.; Paulin, H.; Mogyoros, J.; Giuliani, M.; Dutcher, J. R., The effect of nanoscale surface curvature on the oligomerization of surface-bound proteins. Journal of The Royal Society Interface 2014, 11 (94), 20130818.

7. Park, S.; Jackman, J. A.; Cho, N.-J., Comparing the Membrane-Interaction Profiles of Two Antiviral Peptides: Insights into Structure-Function Relationship. Langmuir 2019, 35 (30), 9934-9943.

8. Reynwar, B. J.; Illya, G.; Harmandaris, V. A.; Müller, M. M.; Kremer, K.; Deserno, M., Aggregation and vesiculation of membrane proteins by curvature-mediated interactions. Nature 2007, 447 (7143), 461-464. 
9. Subbarao, N. K.; Parente, R. A.; Szoka, F. C.; Nadasdi, L.; Pongracz, K., The pHdependent bilayer destabilization by an amphipathic peptide. Biochemistry 1987, 26 (11), 2964-2972.

10. Huang, R. T. C.; Wahn, K.; Klenk, H. D.; Rott, R., Fusion between cell membrane and liposomes containing the glycoproteins of influenza virus. Virology 1980, 104 (2), 294-302.

11. Skehel, J. J.; Bayley, P. M.; Brown, E. B.; Martin, S. R.; Waterfield, M. D.; White, J. M.; Wilson, I. A.; Wiley, D. C., Changes in the conformation of influenza virus hemagglutinin at the $\mathrm{pH}$ optimum of virus-mediated membrane fusion. Proceedings of the National Academy of Sciences 1982, 79 (4), 968.

12. Kaiser, E. T.; Kezdy, F. J., Amphiphilic secondary structure: design of peptide hormones. Science 1984, 223 (4633), 249.

13. Kyte, J.; Doolittle, R. F., A simple method for displaying the hydropathic character of a protein. Journal of Molecular Biology 1982, 157 (1), 105-132.

14. Wiedman, G.; Wimley, W. C.; Hristova, K., Testing the limits of rational design by engineering $\mathrm{pH}$ sensitivity into membrane-active peptides. Biochimica et Biophysica Acta (BBA) - Biomembranes 2015, 1848 (4), 951-957.

15. Nicol, F.; Nir, S.; Szoka, F. C., Jr., Orientation of the pore-forming peptide GALA in POPC vesicles determined by a BODIPY-avidin/biotin binding assay. Biophysical journal 1999, 76 (4), 2121-2141.

16. Nir, S.; Nicol, F.; Szoka, F. C., Surface aggregation and membrane penetration by peptides: relation to pore formation and fusion. Molecular Membrane Biology 1999, 16 (1), 95-101.

17. Ellens, H.; Bentz, J.; Szoka, F. C., pH-Induced destabilization of phosphatidylethanolamine-containing liposomes: role of bilayer contact. Biochemistry 1984, 23 (7), 1532-1538.

18. Deplazes, E.; Tafalla, B. D.; Cranfield, C. G.; Garcia, A., Role of Ion-Phospholipid Interactions in Zwitterionic Phospholipid Bilayer Ion Permeation. The Journal of Physical Chemistry Letters 2020, 6353-6358.

19. Marcus, Y., Thermodynamics of solvation of ions. Part 5.-Gibbs free energy of hydration at 298.15 K. Journal of the Chemical Society, Faraday Transactions 1991, 87 (18), 2995-2999.

20. Mio, K.; Sato, C., Lipid environment of membrane proteins in cryo-EM based structural analysis. Biophys Rev 2018, 10 (2), 307-316.

21. Brzustowicz, M. R.; Brunger, A. T., X-ray scattering from unilamellar lipid vesicles. Journal of Applied Crystallography 2005, 38 (1), 126-131.

22. Eicher, B.; Heberle, F. A.; Marquardt, D.; Rechberger, G. N.; Katsaras, J.; Pabst, G., Joint small-angle $X$-ray and neutron scattering data analysis of asymmetric lipid vesicles. Journal of Applied Crystallography 2017, 50 (2), 419-429.

23. Huang, C.; Mason, J. T., Geometric packing constraints in egg phosphatidylcholine vesicles. Proceedings of the National Academy of Sciences 1978, 75 (1), 308.

24. Smolentsev, N.; Lütgebaucks, C.; Okur, H. I.; de Beer, A. G. F.; Roke, S., Intermolecular Headgroup Interaction and Hydration as Driving Forces for Lipid Transmembrane Asymmetry. Journal of the American Chemical Society 2016, 138 (12), 4053-4060.

25. Cranfield, C. G.; Berry, T.; Holt, S. A.; Hossain, K. R.; Le Brun, A. P.; Carne, S.; Al Khamici, H.; Coster, H.; Valenzuela, S. M.; Cornell, B., Evidence of the Key Role of $\mathrm{H} 3 \mathrm{O}+$ in Phospholipid Membrane Morphology. Langmuir 2016, 32 (41), 10725-10734. 
26. Deplazes, E.; Poger, D.; Cornell, B.; Cranfield, C. G., The effect of hydronium ions on the structure of phospholipid membranes. Physical Chemistry Chemical Physics 2018, 20 (1), 357-366.

27. Deplazes, E.; Sarrami, F.; Poger, D., Effect of H3O+ on the Structure and Dynamics of Water at the Interface with Phospholipid Bilayers. The Journal of Physical Chemistry B 2020, 124 (8), 1361-1373. 